\title{
Exploiting UAV as NOMA based Relay for Coverage Extension
}

\author{
Syeda Kanwal Zaidi ${ }^{1}$, Syed Faraz Hasan ${ }^{1}$, Xiang Gui ${ }^{1}$, Nazmul Siddique ${ }^{2}$, and Salmiah Ahmad ${ }^{3}$ \\ ${ }^{1}$ School of Engineering and Advanced Technology, Massey University, New Zealand \\ ${ }^{2}$ School of Computing and Intelligent Systems, Ulster University, UK \\ ${ }^{3}$ Department of Electrical and Computer Engineering, King Abdul Aziz University, Jeddah, KSA \\ Correspondance: k.zaidi@massey.ac.nz
}

\begin{abstract}
Unmanned aerial vehicles (UAVs) aided communication has acquired research interest in many civilian and military applications. The use of UAV as base stations and as aerial relays to improve coverage of existing cellular networks is prevalent in current literature. Along with this, a few studies have proposed the use of non-orthogonal multiple access (NOMA) in UAV communications. In this paper, we propose a network where a ground user and an aerial UAV relay is accessed using NOMA, where the UAV acts as decode-and-forward (DF) relay to extend the coverage of source. The performance of the proposed model is shown by evaluating outage behaviour for different transmit power and fading environments with Monte Carlo simulations. System throughput of proposed network appears to be better than orthogonal multiple access (OMA) based equivalent network. The results show that with an adequate height of the UAV NOMA based relay, quality of service (QoS) of cell edge user is satisfactory.
\end{abstract}

Index Terms-Unmanned aerial vehicles (UAVs), nonorthogonal multiple access (NOMA), relaying

\section{INTRODUCTION}

Unmanned Aerial vehicles (UAVs), commonly known as drones, have gained popularity in the last few years in a wide range of applications including parcel delivery, online streaming, traffic monitoring, disaster management, agriculture and farming. In most of the scenarios, these UAVs are deployed as low altitude platforms (LAPs) with the ability of autonomously or remotely operated, with the aim of providing UAV-assisted communication. Recently, UAVs have received significant recognition by academia, research industry and government bodies to aid wireless communications by providing ubiquitous coverage and promote information dissemination [1]. For future wireless communications, these low cost devices with high mobility are expected to provide wireless connectivity in several use cases for example, as a substitute of fixed base station (BS) in case of natural disasters, as an adhoc base station to offload data in crowded networks, as an on demand relay to extend the coverage of existing network when there is severe shadowing between users and the base station. All these mentioned scenarios are applicable to beyond fifth-generation (B5G) networks and UAVs stand as as strong candidate to be utilised in B5G networks.

Non-orthogonal multiple access (NOMA) is an essential technology for the radio access design of $5 \mathrm{G}$ cellular networks.

978-1-5386-3531-5/17/\$31.00 (C) 2017 IEEE
Power-domain NOMA works on the concept of assigning varying power levels to users within the same frequency and time block. Unlike serving the users in orthogonal resource block in orthogonal multiple access (OMA), several research works demonstrated that NOMA improves the spectral efficiency and throughput of the system by serving users non-orthogonally [2]. In NOMA, the users are served together based on their distinct channel conditions and different quality of service (QoS) requirements. Since UAVs have distinct communication characteristics than ground users which are mentioned in Section II, NOMA appears to be a great fit to access ground users and UAV together. This research paper highlights the existing research works where UAV communication has been considered to aid existing cellular networks for improved coverage. Furthermore, a comprehensive overview of the existing works on NOMA based UAV communication is also given. We introduce the concept of ground-aerial NOMA where a ground user and a UAV are served together non-orthogonally based on their unique channel characteristics. In the proposed network, UAV acts as a relay to extend the coverage of source and serve a cell-edge user. With Monte Carlo simulations, we evaluate the performance of the network by evaluating outage behaviour at serviced ground users. We also compare the system throughput of the proposed system with OMA based network and show that our proposed network is superior in performance than OMA based network.

\section{UAVS IN WIRELESS COMMUNICATION}

The ever increasing network connected devices such smartphones, smart-tablets, internet-of-things connected sensors emboss a huge network traffic on wireless networks which then needs to be fueled up with advance technologies to cater the data traffic demand. There has been several new research ventures introduced for example, full duplexing, multiple input multiple output (MIMO), device-to device (D2D) communications, milli meter wave communication, NOMA to meet B5G network goals. To meet the similar objectives, UAVs have also been introduced in several research works to improve the network coverage and provide seamless connectivity to the devices where infrastructure is not present or been demolished because of natural disasters. In [1], a high level UAVaided network architecture is explained to highlight the main channel characteristics, design considerations and available 


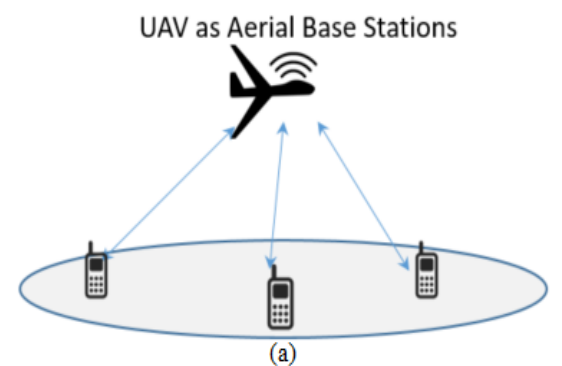

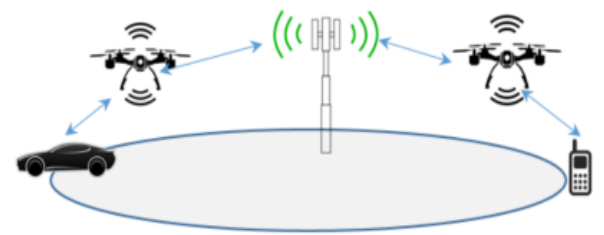

(b)

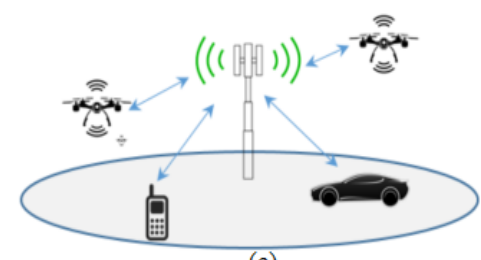

(c)

Fig. 1. UAV as a) Aerial base station b) Aerial relay c) Cellular connected user.

new opportunities in future networks. Similarly, a UAV based aerial wireless network (ABSOLUTE project) is reported in [3], where the LTE-Advanced (LTE-A) BS are mounted on the LAPs to provide wireless connectivity and coverage in the case of unexpected disastrous events.

Here, we have summarised the related literature in three categories. The first category covers the use of UAVs as aerial base stations, the second explains the research work where UAVs are considered as relays for network coverage extension and the third elaborates the idea of UAV as a cellular connected device. Fig. 1 illustrates the typical usage of UAVs in recent research works which is further explained in the following.

\section{A. UAVs as Aerial Base Stations}

Several research works have envisioned the UAVs as flying base stations in heterogeneous cellular networks to provide greater flexibility and scalability in dynamic scenarios and overcome the issues and challenges of existing networks [3], [4], [5], [6], [7]. A concept to provide multiple BS in a macro hot spot emergency area with multiple flying drones is introduced in [8]. It is shown that with the help of game theoretic distributed model based mobility of drones in a specified area helps to provide flexible coverage with improved network throughput. Unlike static user scenario, based on requirement and mobility of users, authors in [9] proposed a framework of self-organising of flying BS (FlyBSs) named as FlyRAN (flying radio access network) to meet users connectivity demand along with the discussion on the implementation and operational issues of FlyBSs. This research also reveals that the FlyBSs are efficient in terms of channel quality and throughput.

Exploiting NOMA in communicating with users improves system throughput, supports massive connectivity, reduces latency, and provides superior spectral efficiency. Sharma et. al deployed a flying BS which employs NOMA to serve two users on the ground [10]. To give insights on the performance, the authors derived dominant conditions, which are based on channel characteristics and UAVs trajectory to guarantee better performance than OMA. As the drones have their unique physical characteristics like altitude from the ground, userfairness maximisation as a function of drone's altitude is studied in [11]. The results revealed that NOMA in drone small cells improve sum rate of the system along with fairness between the users. An extension of similar concept is done in [12] where the energy efficiency along with spectral efficiency is improved by considering fixed and optimised altitude of NOMA based UAV BS. Another approach is taken in [13] by considering a multi-user communication system, in which a single-antenna UAV-BS serves a large number of ground users by employing NOMA. The max-min rate optimization problem is formulated under total power, total bandwidth, UAV altitude, and antenna beam width constraints. Instead of optimising a single parameter, a joint optimisation of the parameters is performed to observe gain in the system rate. Unlike the aforementioned works where a single antenna UAV is used, in [14], a MIMO-NOMA downlink communication scenario in which a UAV equipped with $K$ antennas is communicating with multiple users equipped with $N$ antennas. The performance of the system is observed by deriving outage probabilities of users and diversity order. A stochastic geometry based analysis of UAV-aided cellular networks is done in terms of coverage probability in urban environments to find the optimal height and density of UAVs for maximising coverage [15].

\section{B. UAVs as Relays}

Various research studies have been performed where UAVs are utilised in forming wireless relay networks for data collection and data ferrying. Close to the concept of buffer-aided relay, a three step approach to carry and forward the data using UAV relay in delay-tolerant network is proposed in [16]. Since UAV's trajectory is an important mobility characteristic, its impact on various relaying scenarios is studied in [17], [18]. It is shown in [19] that circular trajectory of UAV relay caused strict limitation on outage probability and data rate of ground users, hence a variable rate approach is provided to optimise achievable performance. As mentioned earlier, the altitude of UAV plays a vital role in deriving the achievable performance, the research work in [20] derived the optimal altitude of amplify and forward (AF) and decode and forward (DF) UAVs for reducing total power loss, outage and improve bit error rate. Along similar lines, in [21] a cooperative relay based UAV network is considered where finite mixture with expectation-maximization algorithm is utilized to derive a sim- 


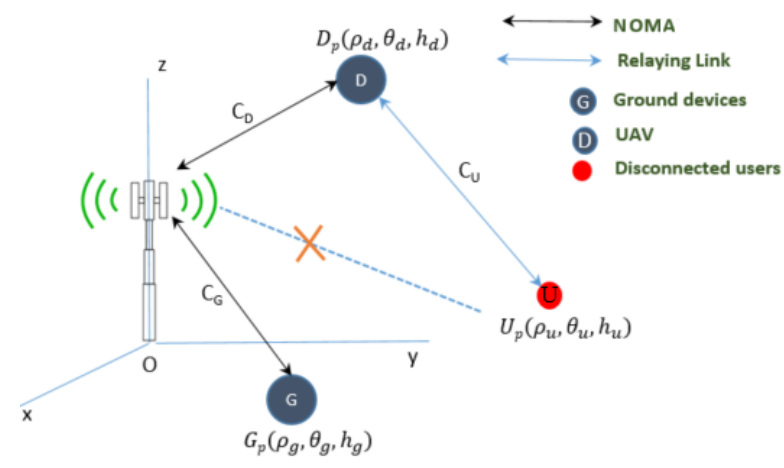

Fig. 2. System Model.

ple approximate expression for the probability density function of the achievable bit rate assuming adaptive M-ary quadrature amplitude modulation (M-QAM). The outage probability and the average achievable bit rate of a cooperative multi-carrier system are derived over generalized Gaussian-Finite-Mixture fading channels. Considering the dynamic mobility of drones, a UAV enabled system is investigated in [22]. Throughput maximisation is done by optimising transmit power, trajectory, speed and initial/final location of UAV.

\section{UAVs as Cellular connected users}

UAVs these days use unlicensed band (for e.g, ISM 2.4 $\mathrm{GHz}$ ) to perform simple point to point communication. As this band is of low data rate, insecure and difficult to manage and the number of UAV applications are expected to grow in future, it is important to characterise significant mechanisms for UAV to ground communications. Cellular connected UAVs is one of the predicted solution to achieve application goals [23] . There are very few works which have investigated UAV as a potential user in the future wireless networks. While the benefits that UAV brings as a flying base station or mobile relay are very much explored, its need of connectivity as a cellular user has not been studied in detail, which is the focus of this paper.

\section{Proposed Model}

In this section, we propose UAV as a cellular connected relay to extend the coverage of BS exploiting NOMA. Our aim is to apply NOMA on two different set of users, one is on ground and the other is aerial user which is a UAV. The UAV acts as a DF relay to forward the data to the cell edge user to extend the coverage of the BS. This is different than ground NOMA where the users who receive NOMA message are present on the ground. We investigate the outage performance of ground users, evaluate impact of height of UAV on the outage under different transmit power and channel conditions.

Consider a network where two ground users $G$ and $U$ needs to be served by a source node $(S)$, where $G$ is close to $S$ and $U$ is located at the edge of the cell. To transmit data,
$S$ utilise a drone user $D$ to relay information to $U$. In a cylindrical coordinate system, we assume that $S$ is located at origin and the locations of $G, U$, and $D$ are expressed as $G_{p}\left(\rho_{G}, \theta_{G}, h_{G}\right), U_{p}\left(\rho_{U}, \theta_{U}, h_{U}\right)$ and $D_{p}\left(\rho_{D}, \theta_{D}, h_{D}\right)$ respectively. The distance between $S$ and $D, S$ and $G$, and $D$ and $U$ are expressed as $\mathcal{D}_{D}, \mathcal{D}_{G}$, and $\mathcal{D}_{U}$ respectively which can be calculated by euclidean norm. Throughout the paper, we consider $D$ as a decode-and-forward half duplex relay, where $S$ transmits symbol $x_{U}$ to $D$ in the first phase, which in second phase, is transmitted by $D$ to $U$ after decoding. All nodes are considered to be single-antenna nodes.

\section{A. Channel Model}

We assume that all the channel links exhibit large-scale path loss and statistically independent frequency non-selective quasi static fading. For $S \leftrightarrow G$ link, Rayleigh fading is considered [2]. If the channel exhibits Rayleigh fading then power of the channel $C_{G}$ follows exponential distribution expressed as

$$
f_{\left|C_{G}\right|^{2}}\left(y ; \mu_{G}\right)=\frac{1}{\mu_{G}} e^{-\frac{y}{\mu_{G}}}, \quad \forall y \geq 0,
$$

where $\mu_{G}$ is the mean parameter. For $S \leftrightarrow D$ and $D \leftrightarrow$ $U$ channel, Nakagami fading is assumed [24] and hence the power of the channels $C_{D}$ and $C_{U}$ follows Gamma distribution is given as

$$
f_{\left|C_{D}\right|^{2},\left|C_{U}\right|^{2}}\left(y ; m, \Omega_{d}\right)=\frac{m_{d}{ }^{m_{d}} y^{m_{d}-1}}{\Omega_{d}{ }^{m_{d}} \Gamma\left(m_{d}\right)} e^{-\frac{m_{d}}{\Omega_{d}} y}, \forall y>0,
$$

where, $m_{d}$ and $\Omega_{d}$ are Nakagami shape and spread control parameters respectively, and $\Gamma\left(m_{d}\right)$ is the Gamma function.

\section{B. Information Theoretic}

At first, $S$ composes a NOMA signal with fixed power allocation for $G$ and $D$ to transmit to $G$ and $D$ respectively, with $\mathcal{P}_{S}$ as the transmit power of $S . \mathcal{P}_{G}$ and $\mathcal{P}_{D}$ are power allocation coefficients for $G$ and $D$ respectively, and $\mathcal{P}_{G}^{2}+\mathcal{P}_{D}^{2}=1$. Since $G$ is located closely to $S, \mathcal{P}_{G}$ is allocated a smaller value than $\mathcal{P}_{D}$, based on the principles of NOMA [2]. The signal received by $G$ and $D$ respectively is expressed as

$$
\mathcal{Y}_{G}=\frac{\sqrt{\mathcal{P}_{S}} \mathcal{P}_{G} x_{G} C_{G}}{\mathcal{D}_{G}^{\alpha_{G}}}+\frac{\sqrt{\mathcal{P}_{S}} \mathcal{P}_{D} x_{U} C_{G}}{\mathcal{D}_{G}^{\alpha_{D}}}+\mathcal{N}_{G},
$$

and

$$
\mathcal{Y}_{D}=\frac{\sqrt{\mathcal{P}_{S}} \mathcal{P}_{D} x_{U} C_{D}}{\mathcal{D}_{D}^{\alpha_{D}}}+\frac{\sqrt{\mathcal{P}_{S}} \mathcal{P}_{G} x_{G} C_{D}}{\mathcal{D}_{D}^{\alpha_{D}}}+\mathcal{N}_{D},
$$

where, $x_{G}$ and $x_{U}$ are the transmit symbols for $G$ and $U$ receptively, $\mathcal{N}_{G} \sim \mathcal{C N}\left(0, \sigma_{G}^{2}\right)$ and $\mathcal{N}_{D} \sim \mathcal{C N}\left(0, \sigma_{D}^{2}\right)$ denote the additive white Gaussian noise (AWGN) with variance $\sigma_{G}^{2}$ and $\sigma_{D}^{2}$ at $G$ and $D$ respectively. To express power-law path loss model, $\alpha_{G}$ and $\alpha_{D}$ represent the path loss exponent for $G$ and $D$ respectively. 


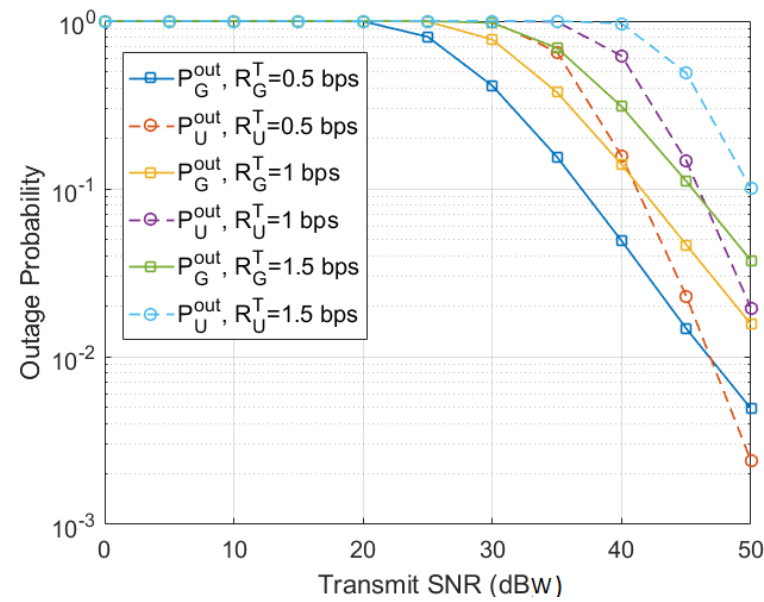

Fig. 3. Outage Probability at $G$ and $U$ with different data rates with $\mathcal{P}_{D}^{\prime}=$ $20 \mathrm{~dB}$.

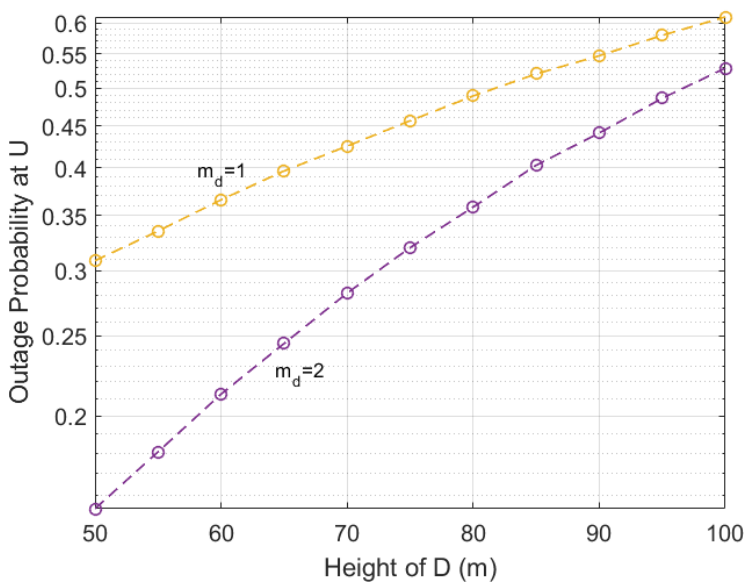

Fig. 4. Outage Probability at $U$ vs. height of $D$ with different values of $m_{d}$, $R_{U}^{T}=0.5$ bps.

$G$ invokes successive interference cancellation (SIC) and decodes $x_{U}$ first which is transmitted by higher power, followed by $x_{G}$ which is transmitted with less power. Taking $\omega_{k}=\frac{\mathcal{P}_{S}}{\sigma_{k}^{2}}$, where $k \epsilon\{G, D\}$, signal to interference and noise ratio (SINR) at $G$ to decode $x_{U}$ and $x_{G}$ is given as

$$
\begin{gathered}
\operatorname{SIN} R_{G}^{x_{U}}=\frac{\omega_{G}\left|C_{G}\right|^{2} \mathcal{P}_{D}^{2}}{\omega_{G}\left|C_{G}\right|^{2} \mathcal{P}_{G}^{2}+\mathcal{D}_{G}^{\alpha_{G}}}, \\
\operatorname{SINR} R_{G}^{x_{G}}=\frac{\omega_{G}\left|C_{G}\right|^{2} \mathcal{P}_{G}^{2}}{\mathcal{D}_{G}^{\alpha_{G}}},
\end{gathered}
$$

For $D, x_{U}$ is received with higher power so it straight away decodes it with following SINR

$$
\operatorname{SIN} R_{D}^{x_{U}}=\frac{\omega_{D}\left|C_{D}\right|^{2} \mathcal{P}_{D}^{2}}{\omega_{D}\left|C_{D}\right|^{2} \mathcal{P}_{G}^{2}+\mathcal{D}_{D}^{\alpha_{D}}},
$$

Once $x_{U}$ is decoded, it is forwarded to $U$ by $D$ with power $\mathcal{P}_{D}^{\prime}$. The signal receieved by $U$ is expressed as

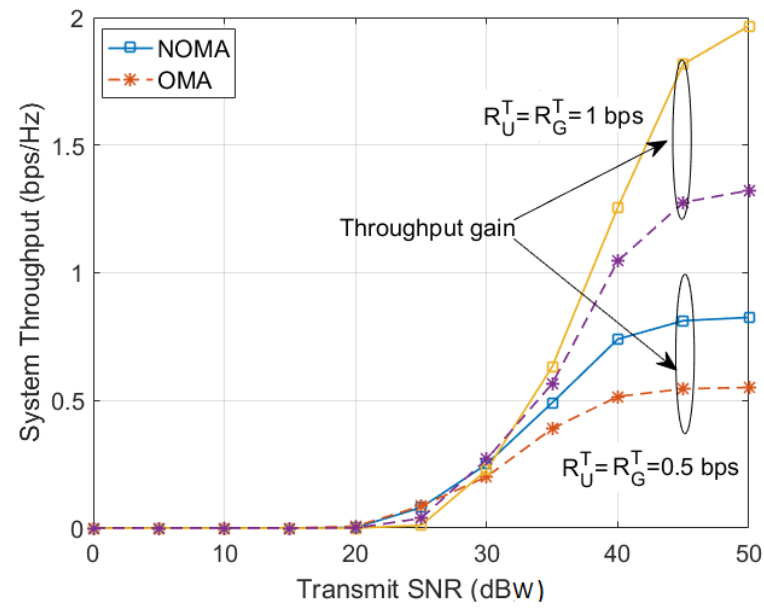

Fig. 5. System Throughput vs. transmit power with different data rates.

$$
\mathcal{Y}_{U}=\frac{\sqrt{\mathcal{P}_{D}^{\prime}} x_{U} C_{U}}{\mathcal{D}_{U}^{\alpha_{D}}}+\mathcal{N}_{U}
$$

Taking $\omega_{U}=\frac{\mathcal{P}_{D}^{\prime}}{\sigma_{U}^{2}}$, SINR at $U$ to decode $x_{U}$ is given as

$$
\operatorname{SINR} R_{U}^{x_{U}}=\frac{\omega_{U}\left|C_{U}\right|^{2}}{\mathcal{D}_{U}^{\alpha_{D}}}
$$

\section{Performance Evaluation}

The performance of the proposed model is characterised by evaluating the outage probability of the user $G$ and $U$. Outage probability is defined as the probability when the observed rate is less than the threshold data rate required by the users to meet quality of service $(Q o S)$ or equivalently when the received SINR is below the target SINR, expressed as follows.

$$
\mathbb{P}_{G}^{\text {out }}=\operatorname{Pr}\left(S I N R_{G}^{x_{G}}<S I N R_{G}^{T}, S I N R_{G}^{x_{U}}<S I N R_{U}^{T}\right)
$$

where, $S I N R_{G}^{T}=2^{R_{G}^{T}}-1$, and $R_{G}^{T}$ is the threshold data rate for $G$.

Since $U$ receives $x_{U}$ by $D, \mathbb{P}_{U}^{\text {out }}$ is expressed as

$$
\mathbb{P}_{U}^{\text {out }}=\operatorname{Pr}\left(S I N R_{D}^{x_{U}}<S I N R_{U}^{T}, S I N R_{U}^{x_{U}}<S I N R_{U}^{T}\right)
$$

where, $S I N R_{U}^{T}=2^{R_{U}^{T}}-1$, and $R_{U}^{T}$ is the threshold data rate for $U$.

\section{A. Simulation Setup}

We consider that $S$ is located at $(0,0,0) . G, D$ and $U$ are located at $G_{p}\left(30, \frac{\pi}{4}, 0\right), D_{p}\left(50, \frac{\pi}{4}, 50\right)$, and $U_{p}\left(100, \frac{\pi}{4}, 0\right)$, unless otherwise stated. The path loss exponent for UAV links $\alpha_{D}$ is taken as 2.05 [24] and path loss exponent for ground users $G$ is taken as 2.7 (for urban wireless network). For simplicity, we assume normalised unit noise at all ends and $\Omega_{d}=\mu_{G}=1$. Fading parameter $m_{d}$ for nakagami fading channel is taken initially as 2 , but altered in some results as stated. Power allocation factors are fixed as $\mathcal{P}_{D}^{2}=0.8$ and $\mathcal{P}_{G}^{2}=0.2$, and $R_{U}^{T}$ is chosen to satisfy NOMA protocol 
$\mathcal{P}_{D}^{2}-\mathcal{P}_{G}^{2} R_{U}^{T}>0$ [2]. Further, for simulations we ran the network for $10 \mathrm{k}$ steps to achieve steady state results.

\section{B. Numerical Results}

Fig. 3 plots the outage probability $\mathbb{P}^{o u t}$ of $G$ and $U$ against different values of transmit source power $\mathcal{P}_{S}$. The results are obtained by the comparison stated in (10) and (11) with threshold data rate as specified in Fig.3. The results show that with increasing value of threshold data rate, outage at both user increases and also the increase in transmit SNR reduces the outage at $G$ and $U$. Since $D$ transmits with fixed power in this case, the outage at $U$ is varied by the outage of $x_{U}$ at drone. The increase in $\mathcal{P}_{S}$ decreases outage at $D$ which in turn reduces outage at $U$. One way of reducing the outage at $U$ is to increase transmit power of $D$ but the constraints on size, weight, and power (SWAP) of small drones makes the transmit power limited.

Another observation is depicted in Fig.4, where the outage at $U$ is illustrated by varying the height of $D$. The outage at $U$ increases with the increased height of drone hence it is important to use drone as relays with the minimum altitude. The restrictions on the height of drones in certain region do not allow drones to be used below a specific height, therefore specifying a minimum height of drone relay is crucial. It can also be observed from Fig.4 that for higher value of $m_{d}$, the outage probability decreases.

Fig. 5 plots system throughput achieved by proposed system compared to OMA based system with different threshold data rates. It is evident from the figure that NOMA based model offers superior throughput than OMA based network where each user is accessed individually in different time slot and with different frequency.

\section{CONCLUSiON}

In this paper, we have provided a brief overview of unmanned aerial vehicles as base stations and relays. We also highlighted the research works where NOMA is employed in UAV communications. Further, we proposed a NOMA based UAV relay to forward the data to a cell edge user. We specifically exploited NOMA with a ground user and aerial relay to extend the coverage of base station to a cell edge user. Simulation results show that with high transmit power, quality of service at a cell edge user is achievable using an intermediate NOMA based aerial UAV relay. Also, the proposed system offers superior throughput than OMA based network. Future extension of this work is to optimise the power allocation coefficients and height of the drone jointly to achieve maximum throughput.

\section{REFERENCES}

[1] Y. Zeng, R. Zhang, and T. J. Lim, "Wireless communications with unmanned aerial vehicles: opportunities and challenges," arXiv preprint arXiv:1602.03602, 2016.

[2] A. Li, Y. Lan, X. Chen, and H. Jiang, "Non-orthogonal multiple access (noma) for future downlink radio access of 5g," China Communications, vol. 12, no. Supplement, pp. 28-37, December 2015.
[3] S. Chandrasekharan, K. Gomez, A. Al-Hourani, S. Kandeepan, T. Rasheed, L. Goratti, L. Reynaud, D. Grace, I. Bucaille, T. Wirth et al., "Designing and implementing future aerial communication networks," IEEE Communications Magazine, vol. 54, no. 5, pp. 26-34, 2016.

[4] I. Bor-Yaliniz and H. Yanikomeroglu, "The new frontier in ran heterogeneity: Multi-tier drone-cells," IEEE Communications Magazine, vol. 54, no. 11, pp. 48-55, 2016.

[5] B. Galkin, J. Kibilda, and L. A. DaSilva, "Deployment of uav-mounted access points according to spatial user locations in two-tier cellular networks," in Wireless Days (WD), 2016. IEEE, 2016, pp. 1-6.

[6] R. I. Bor-Yaliniz, A. El-Keyi, and H. Yanikomeroglu, "Efficient 3-d placement of an aerial base station in next generation cellular networks," in Communications (ICC), 2016 IEEE International Conference on. IEEE, 2016, pp. 1-5.

[7] B. Van der Bergh, A. Chiumento, and S. Pollin, "Lte in the sky: trading off propagation benefits with interference costs for aerial nodes," IEEE Communications Magazine, vol. 54, no. 5, pp. 44-50, 2016.

[8] A. Fotouhi, M. Ding, and M. Hassan, "Flying drone base stations for macro hotspots," IEEE Access, vol. 6, pp. 19530-19539, 2018.

[9] Z. Becvar, M. Vondra, P. Mach, J. Plachy, and D. Gesbert, "Performance of mobile networks with uavs: Can flying base stations substitute ultradense small cells?" in European Wireless 2017; 23th European Wireless Conference; Proceedings of. VDE, 2017, pp. 1-7.

[10] P. K. Sharma and D. I. Kim, "Uav-enabled downlink wireless system with non-orthogonal multiple access," in Globecom Workshops (GC Wkshps), 2017 IEEE. IEEE, 2017, pp. 1-6.

[11] M. F. Sohail and C. Y. Leow, "Maximized fairness for noma based drone communication system," in Communications (MICC), 2017 IEEE 13th Malaysia International Conference on. IEEE, 2017, pp. 119-123.

[12] M. F. Sohail, C. Y. Leow, and S. Won, "Non-orthogonal multiple access for unmanned aerial vehicle assisted communication," IEEE Access, vol. 6, pp. 22 716-22 727, 2018.

[13] Y. Liu, Z. Qin, Y. Cai, Y. Gao, G. Y. Li, and A. Nallanathan, "Uav communications based on non-orthogonal multiple access," arXiv preprint arXiv:1809.05767, 2018.

[14] T. Hou, Y. Liu, Z. Song, X. Sun, and Y. Chen, "Multiple antenna aided noma in uav networks: A stochastic geometry approach," arXiv preprint arXiv:1805.04985, 2018.

[15] L. Zhou, Z. Yang, S. Zhou, and W. Zhang, "Coverage probability analysis of uav cellular networks in urban environments," arXiv preprint arXiv:1805.04404, 2018.

[16] C.-M. Cheng, P.-H. Hsiao, H. Kung, and D. Vlah, "Maximizing throughput of uav-relaying networks with the load-carry-and-deliver paradigm," in Wireless Communications and Networking Conference, 2007. WCNC 2007. IEEE. IEEE, 2007, pp. 4417-4424.

[17] P. Zhan, K. Yu, and A. L. Swindlehurst, "Wireless relay communications with unmanned aerial vehicles: Performance and optimization," IEEE Transactions on Aerospace and Electronic Systems, vol. 47, no. 3, pp. 2068-2085, 2011.

[18] C. Dixon and E. W. Frew, "Optimizing cascaded chains of unmanned aircraft acting as communication relays," IEEE Journal on Selected Areas in Communications, vol. 30, no. 5, pp. 883-898, 2012.

[19] F. Ono, H. Ochiai, and R. Miura, "A wireless relay network based on unmanned aircraft system with rate optimization." IEEE Trans. Wireless Communications, vol. 15, no. 11, pp. 7699-7708, 2016.

[20] Y. Chen, W. Feng, and G. Zheng, "Optimum placement of uav as relays," IEEE Communications Letters, vol. 22, no. 2, pp. 248-251, 2018.

[21] I. Y. Abualhaol and M. M. Matalgah, "Performance analysis of multicarrier relay-based uav network over fading channels," in GLOBECOM Workshops (GC Wkshps), 2010 IEEE. IEEE, 2010, pp. 1811-1815.

[22] Y. Zeng, R. Zhang, and T. J. Lim, "Throughput maximization for uavenabled mobile relaying systems," IEEE Transactions on Communications, vol. 64, no. 12, pp. 4983-4996, 2016.

[23] Y. Zeng, J. Lyu, and R. Zhang, "Cellular-connected uav: Potentials, challenges and promising technologies," arXiv preprint arXiv:1804.02217, 2018.

[24] A. A. Khuwaja, Y. Chen, N. Zhao, M.-S. Alouini, and P. Dobbins, "A survey of channel modeling for uav communications," arXiv preprint arXiv:1801.07359, 2018. 